\title{
The Association Between Hysterectomy and Ovarian Cancer Risk: A Population-Based Record-Linkage Study
}

\author{
Suzanne C. Dixon-Suen, Penelope M. Webb, Louise F. Wilson, Karen Tuesley, \\ Louise M. Stewart, Susan J. Jordan
}

See the Notes section for the full list of authors' affiliations.

Correspondence to: Suzanne C. Dixon-Suen, MEpi, Population Health Department, QIMR Berghofer Medical Research Institute, Locked Bag 2000, Royal Brisbane Hospital, Herston Qld 4029, Australia (e-mail: Suzanne.Dixon@uqconnect.edu.au).

\begin{abstract}
Background: Recent studies have called into question the long-held belief that hysterectomy without oophorectomy protects against ovarian cancer. This population-based longitudinal record-linkage study aimed to explore this relationship, overall and by age at hysterectomy, time period, surgery type, and indication for hysterectomy.

Methods: We followed the female adult Western Australian population (837 942 women) across a 27-year period using linked electoral, hospital, births, deaths, and cancer records. Surgery dates were determined from hospital records, and ovarian cancer diagnoses $(n=1640)$ were ascertained from cancer registry records. We used Cox regression to estimate hazard ratios (HRs) and 95\% confidence intervals (CIs) for the association between hysterectomy and ovarian cancer incidence.

Results: Hysterectomy without oophorectomy $(\mathrm{n}=78$ 594) was not associated with risk of invasive ovarian cancer overall (HR $=0.98,95 \% \mathrm{CI}=0.85$ to 1.11$)$ or with the most common serous subtype $(\mathrm{HR}=1.05,95 \% \mathrm{CI}=0.89$ to 1.23$)$. Estimates did not vary statistically significantly by age at procedure, time period, or surgical approach. However, among women with endometriosis (5.8\%) or with fibroids (5.7\%), hysterectomy was associated with substantially decreased ovarian cancer risk overall ( $\mathrm{HR}=0.17,95 \% \mathrm{CI}=0.12$ to 0.24 , and $\mathrm{HR}=0.27,95 \% \mathrm{CI}=0.20$ to 0.36 , respectively) and across all subtypes.

Conclusions: Our results suggest that for most women, having a hysterectomy with ovarian conservation is not likely to substantially alter their risk of developing ovarian cancer. However, our results, if confirmed, suggest that ovarian cancer risk reduction could be considered as a possible benefit of hysterectomy when making decisions about surgical management of endometriosis or fibroids.
\end{abstract}

Hysterectomy without oophorectomy has been traditionally considered protective against ovarian cancer, supported by a considerable body of older research [summarized in (1-3)]. However, many studies including women diagnosed more recently have not seen a protective association; indeed, some reported modest increases in risk, as seen in a recent metaanalysis (4) and subsequent studies $(5,6)$. This apparent shift could arise from changes in surgical practices or patient characteristics, changes in menopausal hormone therapy (MHT) use, or perhaps improved reporting of oophorectomy status.

\begin{abstract}
Hysterectomy is one of the most common major surgeries among women globally $(7,8)$; understanding the true relationship between hysterectomy alone and ovarian cancer risk is important to inform the current debate over the relative advantages of prophylactically removing ovaries at hysterectomy. Our aim was to explore the association between hysterectomy for benign indications and ovarian cancer risk, overall and by time period, age at surgery, hysterectomy type, and indication for hysterectomy. We examined this question using 45 years of administrative data from the Western Australian Data Linkage System (WADLS) (9).
\end{abstract}




\section{Methods}

\section{Study Population and Linkage}

We conducted a retrospective cohort study including all women on the Western Australian (WA) Electoral Roll (enrollment to vote is compulsory) when electronic records began in 1988, or who registered subsequently. We restricted the cohort to women aged 45 years and younger in 1975, when hysterectomy began to be reliably recorded, to minimize inclusion of women who had hysterectomies, oophorectomies, or cancer diagnoses before observation. Women born from 1930 to 1998 (the last birth-year for which women would reach electoral enrollment age within our observation period) were therefore eligible for the analysis. This cohort was linked with the WA Hospital Morbidity Data Collection (HMDC; 1970-2014), WA Cancer Registry (1982-2015), Midwives Notifications System (MNS; 1980-2013), and WA Births (1950-1979) and Deaths (1988-2015) registers, allowing follow-up from 1988 to mid-2015. The Western Australian Data Linkage Branch linked the datasets using probabilistic linkage matching on identifying and demographic fields and provided de-identified data to the researchers. We excluded women with recorded ovarian cancer, oophorectomy, or hysterectomy for malignancy before their first electoral record $(n=9805)$, or data inconsistencies (implausible birthdates, births after hysterectomy, first electoral record after death, or male-specific codes: $n=1313$ ).

The study received approval from QIMR Berghofer and WA Health Human Research Ethics Committees.

\section{Variable Measurement}

Our main outcome was diagnosis of primary invasive epithelial cancer in the ovary (International Classification of Diseases [ICD]10 code C56), fallopian tube (C57.0), or peritoneum (C48), recorded in the WA Cancer Registry, hereafter referred to collectively as "ovarian cancer." Available data included date and basis of diagnosis, and tumor site, behavior, grade, and morphology. Using morphology International Classification of Diseases for Oncology (ICD-O)-3 codes, we classified cancers as serous, mucinous, endometrioid, clear cell, or other.

The HMDC contains information on all public and private WA hospital separations, including admission and discharge month and year, diagnosis codes (principal, 21 additional), and surgical procedure codes (principal, 10 additional) (see Supplementary Tables 1 and 2, available online, for codes). We used procedure codes to ascertain hysterectomies, oophorectomies, and tubal ligations and diagnosis codes to identify hysterectomy indications and ovarian cancer diagnoses before cancer registry records began.

Birth-year (from electoral records) was provided in 5-year bands to protect anonymity; we derived age by assigning birthdate as the lowermost end. Electoral records include postcode, from which we derived remoteness of residence (major cities, inner regional, outer regional, remote, or very remote) using the Accessibility and Remoteness Index of Australia (ARIA) $(10,11)$, and socio-economic disadvantage quintiles using the SocioEconomic Indexes for Areas (SEIFA) (12), at study entry. We determined parity using the Births Register and MNS, assuming nulliparity at 1950 for women $(n=68218)$ who reached reproductive age before records began.

\section{Statistical Analysis}

We calculated person-time for each woman as the interval between study entry (her first appearance in the available electoral records) and exit, determined as the earliest of: ovarian cancer diagnosis; hysterectomy or unilateral or bilateral oophorectomy if followed within 6 months by ovarian cancer diagnosis (surgery date was then assigned as diagnosis date because we considered these surgeries were likely related to their cancer); or a censoring condition, at which follow-up ceased. Censoring conditions were: all other unilateral or bilateral oophorectomies (including at hysterectomy); hysterectomy for other malignancies (predominantly endometrial); diagnosis of nonepithelial ovarian cancer; death; emigration; or end of observation (June 30, 2015). We calculated exposed and unexposed person-time considering hysterectomy, parity, and tubal ligation as time-varying using the counting process method (13). Thus, person-time from entry until hysterectomy (or until exit, for unexposed women) was considered unexposed; any observed time after hysterectomy (from study entry, for women with prior hysterectomies) was considered exposed.

We performed Cox proportional hazards regression, estimating hazard ratios (HRs) and 95\% confidence intervals (CI) for the association between hysterectomy and ovarian cancer incidence, overall and for different histologic subtypes, using age as the underlying timescale (14). We stratified models by birth decade, and adjusted for age at entry, parity ( $\geq 3$ vs $0-2$ births), and tubal ligation (yes or no). We omitted SEIFA and ARIA from final models because they did not change HRs for the main association. The proportional hazards assumption was confirmed graphically by plotting Schoenfeld residuals against time.

We conducted additional analyses comparing risk associated with hysterectomy performed in three eras (<1990, 1990-2002, $>2002$ ). Time periods were chosen based on trends in MHT use, which may influence the association (15). MHT use decreased in the 1970s then increased again in the 1980s, became widespread during the 1990s, and then declined sharply from 2002 (16), when Women's Health Initiative investigators reported that combined MHT use was associated with adverse health outcomes (17). We examined whether the association varied by age (as a proxy for menopausal status) at hysterectomy, considering under 45 years (mostly premenopausal women), 45-54 years (perimenopausal women), and 55 years or over (mostly postmenopausal women). We compared risk associated with abdominal or vaginal hysterectomy (numbers were insufficient to examine solely laparoscopic hysterectomy). Lastly, we performed analyses considering hysterectomy indication, focusing on three common indications (endometriosis, uterine leiomyoma [fibroids], and genital prolapse).

We conducted sensitivity analyses, including: varying elements of the methods (using years as the timescale); varying the population (to women <45years when hospital [1970] or cancer registry [1982] records began vs applying no age restriction; excluding women entering after 1988 after usual enrollment age [ $>25$ years, therefore women migrating into WA]; examining associations by birth cohort); selecting different comparison groups (estimating associations for later vs earlier hysterectomies in age and period analyses); and restricting the outcome to cancer of the ovary (censoring at fallopian tube or peritoneal cancer diagnosis).

Analyses were performed in SAS 9.4 (SAS Institute Inc, Charlotte, NC). Tests of statistical significance were two-sided and we used an alpha level of $5 \%$ to determine statistical significance; to determine the statistical significance of the hazard ratios in our analysis, we assessed whether the $95 \%$ confidence interval crossed 1.00 .

\section{Results}

We included 837942 women (aged 17-82 [median 30] years at entry, 17-87 [median 50] years at exit) contributing 15738607 
person-years of follow-up. The cohort included 223049 women (26.6\%) who entered in 1988 (median age at entry and exit, 38 and 65 years, respectively) and 614893 women (73.4\%) who entered later (median age at entry and exit, 26 and 45 years, respectively). Overall, 78594 women had a recorded hysterectomy for a benign indication before study exit (28 091 occurred before entry) and contributed 1335989 exposed person-years. There were 81927 tubal ligations before exit. At their study entry, 715329 (85.4\%) women had 0 to 2 children and 122613 (14.6\%) had 3 or more; by study exit, 610857 (72.9\%) had 0 to 2 children, and 227085 (27.1\%) had 3 or more. At entry, 622446 (74.3\%) lived in major cities, $147874(17.6 \%)$ in inner or outer regional, and $60228(7.2 \%)$ in remote or very remote areas; 31.3\% were in the least and $16.9 \%$ in the most disadvantaged SEIFA quintile. There were 48870 (5.8\%) women with a diagnosis of endometriosis (most at hysterectomy), 47407 (5.7\%) with fibroids (15 037 had both conditions), and 55752 (6.7\%) with prolapse. During the study window, 1640 women were diagnosed with invasive epithelial ovarian cancer (including 1020 serous, 125 mucinous, 146 endometrioid, and 90 clear cell cancers). Table 1 presents population characteristics by hysterectomy exposure status.

Hysterectomy was not associated with decreased risk of invasive epithelial ovarian cancer overall $(\mathrm{HR}=0.98,95 \% \mathrm{CI}=0.85$ to 1.11 ) or for the most common serous subtype (HR $=1.05,95 \%$ $\mathrm{CI}=0.89$ to 1.23 ) after adjusting for age, tubal ligation, and parity. Effect estimates were in the protective direction for mucinous ( $\mathrm{HR}=0.55,95 \% \mathrm{CI}=0.28$ to 1.06 ), endometrioid ( $\mathrm{HR}=0.69$, $95 \% \mathrm{CI}=0.41$ to 1.18$)$, and clear cell ( $\mathrm{HR}=0.56,95 \% \mathrm{CI}=0.27$ to 1.16) tumors, but were not statistically significant (Table 2).

Analyses by time period (Figure 1A) showed no association with ovarian cancer overall for hysterectomies performed before 1990 ( $\mathrm{HR}=0.93,95 \% \mathrm{CI}=0.79$ to 1.10$)$ and a slightly positive but statistically nonsignificant association for hysterectomies performed from 1990 to 2002 ( $\mathrm{HR}=1.14,95 \% \mathrm{CI}=0.93$ to 1.40 ). The association for hysterectomies performed after 2002 was inverse $(\mathrm{HR}=0.60,95 \% \mathrm{CI}=0.34$ to 1.06$)$, although not statistically significant. The association did not vary substantively by age at hysterectomy: younger than 45 years $(\mathrm{HR}=0.94,95 \% \mathrm{CI}=0.77$ to 1.15 ); $45-54$ years ( $\mathrm{HR}=1.01,95 \% \mathrm{CI}=0.84$ to 1.21 ); 55 years and older ( $\mathrm{HR}=0.96,95 \% \mathrm{CI}=0.68$ to 1.34 ) (Figure $1 \mathrm{~B}$ ). Results by type of hysterectomy are presented in Supplementary Table 3 (available online). There were too few cancers to conduct period, age, or surgical type analyses by histologic subtype.

Our two a priori approaches to assess whether the association varied by indication were, first, to adjust the main models for the indication(s) for hysterectomy, and second, to examine in a separate model the association between hysterectomy for each indication coded at surgery (a single multilevel variable) and risk, compared to no hysterectomy. Implementing the first approach, we observed that after adjusting models for everdiagnosis of endometriosis or fibroids, estimates of the association decreased for ovarian cancer overall $(\mathrm{HR}=0.65,95 \% \mathrm{CI}=$ 0.56 to 0.75 ) and for each subtype (serous $\mathrm{HR}=0.76,95 \% \mathrm{CI}=$ 0.63 to 0.91 ; mucinous $\mathrm{HR}=0.38,95 \% \mathrm{CI}=0.19$ to 0.75 ; endometrioid $\mathrm{HR}=0.30,95 \% \mathrm{CI}=0.17$ to 0.52 ; clear cell $\mathrm{HR}=0.19,95 \% \mathrm{CI}$ $=0.09$ to 0.41 ) (Table 2). Further adjustment for prolapse did not substantially alter effect estimates.

Implementing our second approach, we constructed a model estimating risk associated with hysterectomy performed for each indication. We observed that, compared to women without hysterectomy (which includes women with or without diagnosis of each indication), estimates were in the direction of slightly increased ovarian cancer risk for hysterectomy for endometriosis without fibroids or prolapse $(\mathrm{HR}=1.18,95 \% \mathrm{CI}=0.71$ to
1.96), or for fibroids without endometriosis or prolapse (HR = $1.10,95 \% \mathrm{CI}=0.79$ to 1.55 ), and apparent slight decreased ovarian cancer risk for hysterectomy for prolapse without endometriosis or fibroids ( $\mathrm{HR}=0.89,95 \% \mathrm{CI}=0.67$ to 1.18 ), although none were statistically significant (Table 3). However, these conditions were associated with ovarian cancer risk irrespective of hysterectomy. Ever-diagnosis of endometriosis was associated with increased ovarian cancer risk $(\mathrm{HR}=2.74,95 \% \mathrm{CI}=2.32$ to 3.22), with particularly strong associations for endometrioid $(\mathrm{HR}=7.42,95 \% \mathrm{CI}=4.80$ to 11.48$)$ and clear cell $(\mathrm{HR}=12.24,95 \%$ $\mathrm{CI}=7.23$ to 20.73 ) cancers (Supplementary Table 4, available online). Ever-diagnosis of fibroids was associated with increased risk overall ( $\mathrm{HR}=1.97,95 \% \mathrm{CI}=1.69$ to 2.30 ), with little variation by subtype (Supplementary Table 4, available online). Everdiagnosis of prolapse was associated with decreased risk overall ( $\mathrm{HR}=0.68,95 \% \mathrm{CI}=0.57$ to 0.80 ), with little variation by subtype except a stronger inverse association for clear cell cancers $(\mathrm{HR}=$ $0.38,95 \% \mathrm{CI}=0.16$ to 0.89 ) (Supplementary Table 4 , available online).

To distinguish the effect of hysterectomy for a particular indication from the effect of the condition itself, we implemented a third approach, stratifying by each condition and repeating our analysis within those strata. We observed that among women ever diagnosed with endometriosis, hysterectomy was associated with substantially decreased ovarian cancer risk (HR $=0.17,95 \% \mathrm{CI}=0.12$ to 0.24 overall) (Table 4 ), with fairly consistent hazard ratios across subtypes (Supplementary Table 5, available online). Similarly, hysterectomy was associated with decreased risk among women with fibroids ( $\mathrm{HR}=0.27,95 \% \mathrm{CI}=$ 0.20 to 0.36 overall) (Table 4), which again was similar across subtypes (Supplementary Table 5, available online). The strong inverse relationship among women with endometriosis or fibroids remained, although slightly attenuated, after excluding women with the other condition (data not shown). Among women without endometriosis, without fibroids, and with neither condition, there was no protective effect of hysterectomy (eg, $\mathrm{HR}=1.15,95 \% \mathrm{CI}=0.98$ to 1.35 overall, among women with neither condition) (Table 4). In women with prolapse, the effect estimate was slightly elevated but not statistically significant ( $\mathrm{HR}=1.24,95 \% \mathrm{CI}=0.91$ to 1.69$)$ (Table 4 ).

Our results were robust to the method used to estimate person-years. Including older women (relaxing or removing the age restriction) yielded similar estimates although some became slightly inverse (Supplementary Table 6, available online), consistent with the hypothesis that including older women increases misclassification of women with prior oophorectomy. Restricting analyses to younger women ( $<45$ years in 1982), or excluding women migrating into WA, made little difference to estimates (Supplementary Table 6, available online). There were no obvious patterns by birth cohort, although numbers were generally too low to draw clear conclusions. Estimates were similar when we repeated analyses defining outcome strictly as cancer of the ovary (ICD code C56), except for a stronger inverse association for mucinous cancers (data not shown).

\section{Discussion}

In this large retrospective study using linked administrative data, we found that hysterectomy was not associated with decreased ovarian cancer risk either overall or for the most common and aggressive form (serous cancer) and that reductions in risk were not observed in earlier time periods. There was a suggestion of decreased risk of nonserous ovarian cancers, which 
Table 1. Demographic and reproductive characteristics of included women, by final hysterectomy status recorded 1970-2015

\begin{tabular}{|c|c|c|c|}
\hline Characteristic & $\begin{array}{l}\text { No hysterectomy } \\
(\mathrm{N}=733832) \\
\text { No. }(\%)\end{array}$ & $\begin{array}{c}\text { Hysterectomy } \\
\text { contributing exposed } \\
\text { time* }(\mathrm{N}=78594) \\
\text { No. }(\%)\end{array}$ & $\begin{array}{c}\text { Hysterectomy contributing } \\
\text { no exposed } \\
\text { time } \dagger(\mathrm{N}=25516) \\
\text { No. }(\%)\end{array}$ \\
\hline \multicolumn{4}{|l|}{ Age at study entry, $\mathrm{y}$} \\
\hline$<30$ & $385380(52.5)$ & $12054(15.3)$ & $5428(21.3)$ \\
\hline 30 to $<40$ & $159300(21.7)$ & $23759(30.2)$ & $7840(30.7)$ \\
\hline 40 to $<50$ & $107808(14.7)$ & $26584(33.8)$ & $8159(32.0)$ \\
\hline 50 to $<60$ & $66378(9.0)$ & $14794(18.8)$ & $3690(14.5)$ \\
\hline 60 to $<70$ & $12870(1.8)$ & $1331(1.7)$ & $373(1.5)$ \\
\hline$\geq 70$ & $2096(0.3)$ & $72(0.1)$ & $26(0.1)$ \\
\hline \multicolumn{4}{|c|}{ Tubal ligation at end of observation } \\
\hline No & $672377(91.6)$ & $62509(79.5)$ & $20677(81.0)$ \\
\hline Yes & $61455(8.4)$ & $16085(20.5)$ & $4839(19.0)$ \\
\hline \multicolumn{4}{|l|}{ Parity at end of observation } \\
\hline 0 & $342192(46.6)$ & $21170(26.9)$ & 8481 (33.2) \\
\hline 1 & $62582(8.5)$ & $6272(8.0)$ & $2176(8.5)$ \\
\hline 2 & $142476(19.4)$ & $19240(24.5)$ & $6268(24.6)$ \\
\hline$\geq 3$ & $186582(25.4)$ & $31912(40.6)$ & $8591(33.7)$ \\
\hline \multicolumn{4}{|l|}{ Endometriosis $\ddagger$} \\
\hline No & $713013(97.2)$ & $59725(76.0)$ & $16334(64.0)$ \\
\hline Yes & $20819(2.8)$ & $18869(24.0)$ & $9182(36.0)$ \\
\hline \multicolumn{4}{|l|}{ Fibroids $\neq$} \\
\hline No & $721137(98.3)$ & $54977(70.0)$ & $14421(56.5)$ \\
\hline Yes & $12695(1.7)$ & $23617(30.0)$ & $11095(43.5)$ \\
\hline \multicolumn{4}{|l|}{ Prolapse $\ddagger$} \\
\hline No & $715643(97.5)$ & $46408(59.0)$ & $20139(78.9)$ \\
\hline Yes & $18189(2.5)$ & $32186(41.0)$ & $5377(21.1)$ \\
\hline \multicolumn{4}{|c|}{ Socio-economic disadvantage score (SEIFA) quintile at study entry§ } \\
\hline 1 (most disadvantaged) & $122758(16.7)$ & $14213(18.1)$ & $4590(18.0)$ \\
\hline 2 & $160028(21.8)$ & $21514(27.4)$ & $6364(24.9)$ \\
\hline 3 & $98783(13.5)$ & $9912(12.6)$ & $3202(12.5)$ \\
\hline 4 & $118065(16.1)$ & $10863(13.8)$ & $3577(14.0)$ \\
\hline 5 (least disadvantaged) & $232575(31.7)$ & $21847(27.8)$ & $7722(30.3)$ \\
\hline \multicolumn{4}{|l|}{ Remoteness at study entry§ } \\
\hline Major cities & $548088(74.7)$ & $55110(70.1)$ & $19248(75.4)$ \\
\hline Inner regional & $63417(8.6)$ & 7880 (10.0) & $2108(8.3)$ \\
\hline Outer regional & $63584(8.7)$ & $8811(11.2)$ & $2074(8.1)$ \\
\hline Remote & $36015(4.9)$ & $4295(5.5)$ & $1212(4.7)$ \\
\hline Very remote & $16772(2.3)$ & $1423(1.8)$ & $511(2.0)$ \\
\hline
\end{tabular}

*These women had a hysterectomy for benign indications before their study exit (their hysterectomies occurred either before their first electoral record, or during the study window) and therefore contribute exposed time to the analysis. Women whose hysterectomy was performed during the study window contribute both unexposed and exposed time. SEIFA = Socio-Economic Indexes for Areas

†These women had a hysterectomy but contribute only unexposed time to the analysis because their hysterectomy occurred at, or after, their study exit (these women predominantly exited because of a bilateral or unilateral oophorectomy performed prior to, or at the time of, their hysterectomy), or was performed for a nonbenign indication ( $n=4632$; at which time follow-up was censored if the woman had not already exited the study).

‡Condition included as a diagnosis during any hospitalizations from 1970 to 2015.

§Percentages do not add to $100 \%$ because of a small proportion of missing data.

was not statistically significant. The association varied minimally by age at surgery or type of hysterectomy. Among women with endometriosis or fibroids (two common indications for this procedure), hysterectomy was associated with substantially decreased ovarian cancer risk.

Although older studies had suggested a protective effect of hysterectomy on ovarian cancer $(18,19)$, almost all recent studies have not shown this $(20,21)$. Our results are consistent with recent findings; we did not see inverse associations even for hysterectomies performed in the 1970s and 1980s. The discrepancy between older and newer studies may arise from better self-reporting of oophorectomy. Many women with documented bilateral oophorectomy report that their ovaries have not been removed (22). It may be that women in older studies, with hysterectomies undertaken further back in time, were less likely to be aware that oophorectomy had been simultaneously performed. Our study avoids this bias by ascertaining oophorectomy using hospital records.

Fluctuations in MHT use may have also influenced associations over time. MHT is prescribed more frequently to women with hysterectomy than those without, and it is associated with modestly increased ovarian cancer risk (23). Two large studies have reported that hysterectomy is associated with slightly increased ovarian cancer risk among ever-users of MHT, but decreased risk among never-users $(24,25)$. Without data on MHT use we could not directly explore its influence, but the slight increased risk associated with hysterectomies in the 1990s, when MHT was common, and reduced risk thereafter, when MHT use 
Table 2. Association between hysterectomy for a benign indication and invasive epithelial ovarian cancer, by histologic subtype*

\begin{tabular}{|c|c|c|c|c|c|}
\hline Adjustment models $\dagger$ & $\begin{array}{l}\text { All invasive ovarian } \\
\text { cancer HR (95\% CI) }\end{array}$ & $\begin{array}{c}\text { Serous } \\
\text { HR ( }(95 \% \text { CI) }\end{array}$ & $\begin{array}{l}\text { Mucinous } \\
\text { HR (95\% CI) }\end{array}$ & $\begin{array}{l}\text { Endometrioid } \\
\text { HR ( } 95 \% \text { CI) }\end{array}$ & $\begin{array}{l}\text { Clear cell } \\
\text { HR (95\% CI) }\end{array}$ \\
\hline \multicolumn{6}{|l|}{ Adjustment 1} \\
\hline No hysterectomy & 1.00 (Referent) & 1.00 (Referent) & 1.00 (Referent) & 1.00 (Referent) & 1.00 (Referent) \\
\hline Hysterectomy & 0.98 (0.85 to 1.11$)$ & 1.05 (0.89 to 1.23$)$ & 0.55 (0.28 to 1.06$)$ & 0.69 (0.41 to 1.18$)$ & 0.56 (0.27 to 1.16$)$ \\
\hline \multicolumn{6}{|l|}{ Adjustment 2} \\
\hline No hysterectomy & 1.00 (Referent) & 1.00 (Referent) & 1.00 (Referent) & 1.00 (Referent) & 1.00 (Referent) \\
\hline Hysterectomy & 0.65 (0.56 to 0.75$)$ & 0.76 (0.63 to 0.91$)$ & 0.38 (0.19 to 0.75$)$ & 0.30 (0.17 to 0.52$)$ & 0.19 (0.09 to 0.41$)$ \\
\hline
\end{tabular}

*Time was censored at hysterectomy for malignancy. Hysterectomy is time-varying. Ovarian cancer includes primary ovarian, fallopian tube, and peritoneal cancer. For each histologic subtype analysis, follow-up time is censored at diagnosis of other subtypes. $\mathrm{HR}=$ hazard ratio; $\mathrm{CI}=\mathrm{confidence} \mathrm{interval}$.

†Models were stratified by decade of birth and adjusted for age at study entry, tubal ligation (yes/no), and parity (3 or more vs 0-2 births) (Adjustment 1), and additionally endometriosis (yes/no) and fibroids (yes/no) (Adjustment 2). Adjusting for genital prolapse (a third common indication for hysterectomy) did not affect the association between hysterectomy and ovarian cancer, therefore this was not included in presented models.

¥As hysterectomy is time-varying, the analysis compares exposed time ("hysterectomy," contributed by women after their hysterectomy) vs unexposed time ("no hysterectomy," contributed by women before their hysterectomy and by women who did not have a recorded hysterectomy). We assigned date of hysterectomy as date of hospital discharge. For the vast majority (99.9\%) of the women in this analysis, admission and discharge dates for hospitalization for hysterectomy were identical (78.9\%) or only one month apart (21.0\%), because recovery in hospital after hysterectomy is typically short and the provided Hospital Morbidity Data Collection data included admission and discharge month and year (not day).
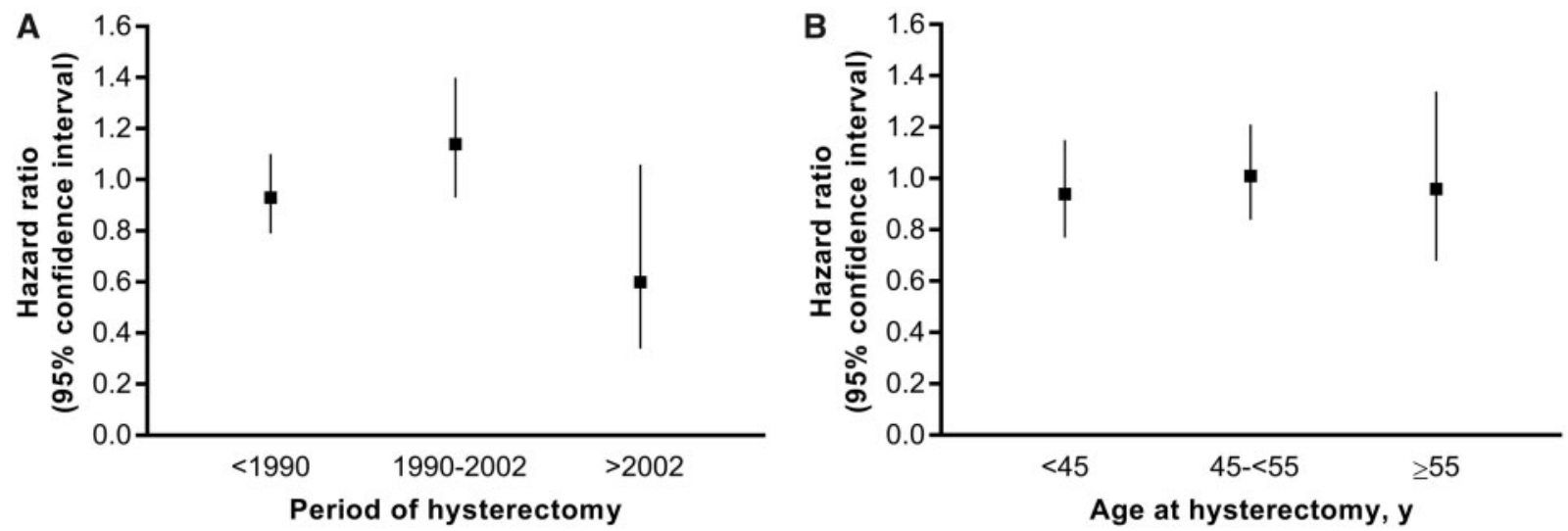

Figure 1. Association between hysterectomy for a benign indication and invasive epithelial ovarian cancer, by (A) period of hysterectomy and (B) age at hysterectomy, compared to no hysterectomy. Time was censored at hysterectomy for malignancy. Hysterectomy is time-varying. Ovarian cancer includes primary ovarian, fallopian tube, and peritoneal cancer. Models were stratified by decade of birth and adjusted for age at study entry, tubal ligation (yes/no), and parity ( 3 or more vs $0-2$ births). In (B), patterns were similar if we assigned birthdate at the electoral birth-year band midpoint.

fell, is consistent with this pattern. However, we had short follow-up and few hysterectomies and cancer diagnoses after 2002.

Some previous studies have suggested that hysterectomy at younger ages particularly ( $<45$ or $<50$ years) may be associated with reduced ovarian cancer risk (4). However, we found little variation in the association by surgery age.

We found some evidence that the association varies by histologic subtype, with no association seen for serous cancers but a suggestion of inverse relationships with nonserous tumors, particularly mucinous and clear cell. These results closely match subtype-specific estimates from a recent pooled analysis of 21 cohorts (6). Some $(5,26-28)$, but not all $(29,30)$, large casecontrol studies have also reported risk reductions for nonserous tumors.

A novel finding of our study was that hysterectomy appeared to substantially reduce risk among women with endometriosis or fibroids (both of which were associated with considerably increased ovarian cancer risk in our population). Few studies have considered indication for hysterectomy. One casecontrol study reported that adjustment for endometriosis, fibroids, and ovarian cysts decreased the odds ratio (OR) for the association between hysterectomy and ovarian cancer (24), as we found. An analysis pooling four case-control studies (2098 case subjects) compared women with and without endometriosis and, consistent with our results, reported that hysterectomy was associated with possible decreased ovarian cancer risk among women with endometriosis $(\mathrm{OR}=0.69,95 \% \mathrm{CI}=$ 0.38 to 1.24 ) but not without ( $\mathrm{OR}=1.02,95 \% \mathrm{CI}=0.86$ to 1.23 ) (31). Use of self-reported endometriosis, consequently including a broader disease spectrum with more misclassification, may explain their weaker association, as self-reported endometriosis is associated with lower ovarian cancer risk than surgically confirmed endometriosis (32). Another study of women with endometriosis found that removal of endometriotic lesions substantially reduced ovarian cancer risk, whereas hysterectomy did not (33). It is possible that the protective effect we observed is primarily due to simultaneous removal of lesions. Fibroids have rarely been assessed in relation to ovarian cancer. One Danish record-linkage study reported that women with fibroids are at increased risk of ovarian cancer (relative risk $[R R]=$ $1.36,95 \% \mathrm{CI}=1.16$ to 1.60 ), particularly endometrioid tumors 
Table 3. Association between hysterectomy for a benign indication and invasive epithelial ovarian cancer, ${ }^{*}$ by indication

\begin{tabular}{lc}
\hline Recorded hysterectomy indication & HR (95\% CI) $\dagger$ \\
\hline No hysterectomy & 1.00 (Referent) \\
Hysterectomy performed for: $¥$ & \\
Endometriosis (not fibroids or prolapse) & 1.18 (0.71 to 1.96$)$ \\
Fibroids (not endometriosis or prolapse) & $1.10(0.79$ to 1.55$)$ \\
Prolapse (not endometriosis or fibroids) & $0.89(0.67$ to 1.18$)$ \\
Fibroids and endometriosis (not prolapse) & $0.80(0.43$ to 1.50$)$ \\
Prolapse and endometriosis (not fibroids) & $0.71(0.32$ to 1.58$)$ \\
Prolapse and fibroids (not endometriosis) & $1.16(0.70$ to 1.93$)$ \\
Prolapse, fibroids, and endometriosis & $0.77(0.32$ to 1.85$)$ \\
Other indications & $0.98(0.82$ to 1.18$)$
\end{tabular}

*Time was censored at hysterectomy for malignancy. Hysterectomy is timevarying. Ovarian cancer includes primary ovarian, fallopian tube, and peritoneal cancer. $\mathrm{HR}=$ hazard ratio; $\mathrm{CI}=$ confidence interval.

†Models were stratified by decade of birth and adjusted for age at study entry, tubal ligation (yes/no), and parity (3 or more vs $0-2$ births).

$\neq$ We created a single time-varying variable for hysterectomy by indication. We defined indication using codes recorded in any of the diagnosis fields at the time of surgery to capture the pathological context of the surgery as completely as possible. Women who had a hysterectomy before study exit contribute exposed time to one of the indication categories from their hysterectomy onward. For each of the following categories, other conditions, such as those coded as uterine disorders or disorders of menstruation, may also have been noted in the hospital record.

Table 4. Association between hysterectomy for a benign indication and invasive epithelial ovarian cancer, ${ }^{*}$ by presence of endometriosis, fibroids, and prolapse $\dagger$

Hysterectomy by presence of benign

gynecological conditions

HR $(95 \%$ CI) $\neq$

Women with endometriosis

No hysterectomy

Hysterectomy

Women without endometriosis

No hysterectomy

Hysterectomy

Women with fibroids

No hysterectomy

Hysterectomy

Women without fibroids

No hysterectomy

Hysterectomy

Women with endometriosis or fibroids

No hysterectomy

Hysterectomy

Women without endometriosis or fibroids

No hysterectomy

Hysterectomy

Women with prolapse

No hysterectomy

Hysterectomy

Women without prolapse

No hysterectomy

Hysterectomy

1.00 (Referent)

0.17 (0.12 to 0.24$)$

1.00 (Referent)

1.09 (0.95 to 1.26$)$

1.00 (Referent)

0.27 (0.20 to 0.36$)$

1.00 (Referent)

1.09 (0.94 to 1.27$)$

1.00 (Referent)

0.26 (0.21 to 0.33$)$

1.00 (Referent)

1.15 (0.98 to 1.35 )

1.00 (Referent)

1.24 (0.91 to 1.69$)$

1.00 (Referent)

0.97 (0.83 to 1.15$)$

*Time was censored at hysterectomy for malignancy. Hysterectomy is timevarying. Ovarian cancer includes primary ovarian, fallopian tube, and peritoneal cancer. $\mathrm{HR}=$ hazard ratio; $\mathrm{CI}=$ confidence interval.

tStrata (presence or absence of each condition) are defined by whether the condition was included as a diagnosis during any hospitalizations 1970-2015. $\ddagger$ Models were stratified by decade of birth and adjusted for age at study entry tubal ligation (yes/no), and parity (3 or more vs 0-2 births).
$(\mathrm{RR}=1.57,95 \% \mathrm{CI}=1.05$ to 2.34 ) (34), a similar although slightly weaker association than we observed. We know of no studies examining the relationship between hysterectomy and ovarian cancer by the presence or absence of fibroids. Our findings among women with these conditions, while intriguing and plausible, were obtained from post hoc subgroup analyses and require replication.

Notable strengths of our study include its large size, population-based design, long-term follow-up, and measurement of exposure and outcome using medical records. Compulsory electoral enrollment allowed us to include virtually all female WA residents. Ascertaining hysterectomy and oophorectomy status using hospital data eliminates recall error, a concern in many previous studies. Misclassification of hysterectomy status is likely to be minimal, because: the HMDC records all surgeries in WA; residents infrequently receive care elsewhere because of geographic isolation (35); we restricted the analysis to younger women at the start of hospital records; and excluding women migrating into WA during observation did not change our findings. Precise information on surgery date allowed accurate classification of exposed time. Reporting diagnoses of malignant solid tumors to the cancer registry is mandatory, so most ovarian cancers after 1982 should be captured.

Our study is not without limitations. We may not have captured exposures or outcomes occurring prior to available hospital records. We mitigated this by restricting to women aged 45 years and younger in 1975 (the start of reliable hysterectomy data). Residual misclassification resulting from unrecorded early hysterectomies (perhaps chiefly among $6.7 \%$ of women without recorded hysterectomy, who entered in 1988 and were aged 30-45 years in 1975) would have reduced the difference between exposed and unexposed groups, attenuating any association. However, restricting our analysis to younger women resulted in similar estimates, suggesting that misclassification was minimal. Our study design limited access to information on some possible confounding factors, which are not usually recorded in administrative data sources. However, the confounders we were able to include had negligible influence on our effect estimates, and confounders that were unrecorded would be expected to have modest effects (for instance, oral contraceptive use is not strongly related to hysterectomy [eg, (36)]). For the same reason, we could not directly assess the effect of any changes in MHT use over time. We compared the association between time periods chosen to reflect different levels of MHT use and observed suggestive differences. MHT use is only moderately associated with ovarian cancer risk (23) but could account for a portion of the difference. Future studies examining this association should incorporate MHT use if possible.

In summary, we conducted a large, population-based record-linkage study using up to 45 years of data for women in Western Australia. Our results for women with endometriosis and fibroids require replication but suggest that hysterectomy may reduce ovarian cancer risk among these women. However, women without these conditions should not be advised that hysterectomy alone will reduce their ovarian cancer risk.

\section{Funding}

The study was supported by an internal grant from QIMR Berghofer Medical Research Institute and a National Health and Medical Research Council of Australia (NHMRC) Program Grant (1073898). SJ and PW were supported by fellowships from the NHMRC. SD was supported by an Australian Government 
Research Training Program scholarship and a QIMR Berghofer Medical Research Institute scholarship.

\section{Notes}

Affiliations of authors: Population Health Department, QIMR Berghofer Medical Research Institute, Brisbane, Australia (SCDS, PMW, LFW, KT, SJJ); School of Public Health, University of Queensland, Brisbane, Australia (SCDS, PMW, KT, SJJ); Health Research and Data Analytics Hub, PHRN Centre for Data Linkage, School of Public Health, Curtin University, Perth, Australia (LMS); Institute for Health Research, University of Notre Dame, Fremantle, Australia (LMS).

The funders had no role in the design of the study; the collection, analysis, and interpretation of the data; the writing of the manuscript; or the decision to submit the manuscript for publication.

The authors have no conflicts of interest to disclose.

The authors wish to thank the staff at the Western Australian Data Linkage Branch and staff and custodians of the Western Australian Hospital Morbidity Data Collection, Cancer Registry, Midwives Notifications System, Births Registry, Deaths Registry, and the Electoral Roll.

\section{References}

1. Runnebaum IB, Stickeler E. Epidemiological and molecular aspects of ovarian cancer risk. J Cancer Res Clin Oncol. 2001;127(2):73-79.

2. Permuth-Wey J, Sellers TA. Chapter 20: Epidemiology of ovarian cancer. In Verma M (ed.), Methods of molecular biology, cancer epidemiology, Vol. 472. 2009. Totowa, NJ: Humana Press.

3. Hunn J, Rodriguez GC. Ovarian cancer: etiology, risk factors, and epidemiology. Clin Obstet Gynecol. 2012;55(1):3-23.

4. Jordan SJ, Nagle CM, Coory MD, Maresco D, Protani MM, Pandeya NA. Has the association between hysterectomy and ovarian cancer changed over time? A systematic review and meta-analysis. Eur J Cancer. 2013;49(17) 3638-3647.

5. Rice MS, Murphy MA, Vitonis AF, et al. Tubal ligation, hysterectomy and epithelial ovarian cancer in the New England Case-Control Study. Int $J$ Cancer. 2013;133(10):2415-2421.

6. Wentzensen N, Poole EM, Trabert B, et al. Ovarian cancer risk factors by histologic subtype: an analysis from the Ovarian Cancer Cohort Consortium. J Clin Oncol. 2016;34(24):2888-2898.

7. Merrill RM. Hysterectomy surveillance in the United States, 1997 through 2005. Med Sci Monit. 2008;14(1):Cr24-Cr31.

8. Gentry-Maharaj A, Glazer C, Burnell M, et al. Changing trends in reproductive/lifestyle factors in UK women: descriptive study within the UK Collaborative Trial of Ovarian Cancer Screening (UKCTOCS). BMJ Open. 2017, 7(3): $\mathrm{e} 011822$.

9. Holman CD, Bass AJ, Rosman DL, et al. A decade of data linkage in Western Australia: strategic design, applications and benefits of the WA data linkage system. Aust Health Rev. 2008;32(4):766-777.

10. Australian Bureau of Statistics. 1216.0-Australian Standard Geographical Classification (ASGC), July 2011. Canberra: Commonwealth of Australia; 2011.

11. Australian Bureau of Statistics. 1216.0-Australian Standard Geographical Classification (ASGC), July 2006. Canberra: Commonwealth of Australia; 2006.

12. Australian Bureau of Statistics. 2033.0.55.001-Census of Population and Housing: Socio-Economic Indexes for Areas (SEIFA), Australia 2011. Canberra: Commonwealth of Australia; 2013.

13. Powell TM, Bagnell ME. Your "Survival" Guide to Using Time-Dependent Covariates. Orlando, FL: SAS Institute Inc; 2012. Document No.: 168.
14. Griffin BA, Anderson GL, Shih RA, Whitsel EA. Use of alternative time scales in Cox proportional hazard models: implications for time-varying environmental exposures. Stat Med. 2012;31(27):3320-3327.

15. Yang HP, Anderson WF, Rosenberg PS, et al. Ovarian cancer incidence trends in relation to changing patterns of menopausal hormone therapy use in the United States. J Clin Oncol. 2013;31(17):2146-2151.

16. Zbuk K, Anand SS. Declining incidence of breast cancer after decreased use of hormone-replacement therapy: magnitude and time lags in different countries. J Epidemiol Community Health. 2012;66(1):1-7.

17. Writing Group for the Women's Health Initiative Investigators. Risks and benefits of estrogen plus progestin in healthy postmenopausal women: principal results from the Women's Health Initiative randomized controlled trial. JAMA. 2002;288(3):321-333.

18. Luoto R, Auvinen A, Pukkala E, Hakama M. Hysterectomy and subsequent risk of cancer. Int J Epidemiol. 1997;26(3):476-483.

19. Risch HA, Marrett LD, Howe GR. Parity, contraception, infertility, and the risk of epithelial ovarian cancer. Am J Epidemiol. 1994;140(7):585-597.

20. Ness RB, Dodge RC, Edwards RP, Baker JA, Moysich KB. Contraception meth ods, beyond oral contraceptives and tubal ligation, and risk of ovarian cancer. Ann Epidemiol. 2011;21(3):188-196.

21. Jordan SJ, Green AC, Whiteman DC, et al. Serous ovarian, fallopian tube and primary peritoneal cancers: a comparative epidemiological analysis. Int Cancer. 2008;122(7):1598-1603.

22. Phipps AI, Buist DS. Validation of self-reported history of hysterectomy and oophorectomy among women in an integrated group practice setting. Menopause. 2009;16(3):576-581.

23. Tsilidis KK, Allen NE, Key TJ, et al. Menopausal hormone therapy and risk of ovarian cancer in the European prospective investigation into cancer and nutrition. Cancer Causes Control. 2011;22(8):1075-1084.

24. Peres LC, Alberg AJ, Bandera EV, et al. Premenopausal hysterectomy and risk of ovarian cancer in African-American women. Am J Epidemiol. 2017;186(1): 46-53.

25. Rice MS, Hankinson SE, Tworoger SS. Tubal ligation, hysterectomy, unilateral oophorectomy, and risk of ovarian cancer in the Nurses' Health Studies. Fertil Steril. 2014;102(1):192-198.e3.

26. Wittenberg J, Cook LS, Rossing MA, Weiss NS, Wittenberg L. Reproductive risk factors for mucinous and non-mucinous epithelial ovarian cancer. Epidemiology. 1999;10(6):761-763.

27. Riman T, Dickman PW, Nilsson S, et al. Risk factors for invasive epithelial ovarian cancer: results from a Swedish case-control study. Am J Epidemiol. 2002;156(4):363-373.

28. Modugno F, Ness RB, Wheeler JE. Reproductive risk factors for epithelial ovarian cancer according to histologic type and invasiveness. Ann Epidemiol. 2001; 11(8):568-574.

29. Nagle CM, Olsen CM, Webb PM, Jordan SJ, Whiteman DC, Green AC Endometrioid and clear cell ovarian cancers: a comparative analysis of risk factors. Eur J Cancer. 2008;44(16):2477-2484.

30. Jordan SJ, Green AC, Whiteman DC, Webb PM. Risk factors for benign, borderline and invasive mucinous ovarian tumors: epidemiological evidence of a neoplastic continuum? Gynecol Oncol. 2007;107(2):223-230.

31. Modugno F, Ness RB, Allen GO, Schildkraut JM, Davis FG, Goodman MT. Oral contraceptive use, reproductive history, and risk of epithelial ovarian cancer in women with and without endometriosis. Am J Obstet Gynecol. 2004;191(3): 733-740.

32. Lee WL, Chang WH, Wang KC, et al. The risk of epithelial ovarian cancer of women with endometriosis may be varied greatly if diagnostic criteria are different: a nationwide population-based cohort study. Medicine. 2015;94(39): e1633.

33. Melin A-S, Lundholm C, Malki N, Swahn M-L, Sparèn P, Bergqvist A. Hormonal and surgical treatments for endometriosis and risk of epithelial ovarian cancer. Acta Obstet Gynecol Scand. 2013;92(5):546-554.

34. Brinton LA, Sakoda LC, Sherman ME, Frederiksen K, Kjaer SK, Graubard BI. Relationship of benign gynecologic diseases to subsequent risk of ovarian and uterine tumors. Cancer Epidemiol Biomarkers Prev. 2005;14(12):2929-2935.

35. Laurvick CL, Semmens JB, Leung YC, Holman CD. Ovarian cancer in Western Australia (1982-1998): trends in surgical intervention and relative survival Gynecol Oncol. 2003;88(2):141-148.

36. Iversen L, Hannaford PC, Elliott AM, Lee AJ. Long term effects of hysterectomy on mortality: Nested cohort study. BMJ. 2005;330(7506):1482 